\title{
Editorial
}

\section{Basic research on high dilutions and possible applications in many fields of knowledge}

\author{
Leoni Villano Bonamin \\ GIRI Editor \\ leonibonamin@gmail.com
}

In this issue (3-4 / 2018) we find five articles that show the great variety of situations in which high dilutions can produce biological effects, whose daily implications are very important. In two articles, the effects of homeopathic preparations on plant development are described, with a positive impact on both agriculture and the preservation of forest areas. The results obtained lead to a reflection on the rational use of high dilutions and the coexistence between the advancement of agricultural productivity - necessary to feed the human and animal population to the satisfaction and the preservation of increasingly scarce natural areas over our planet. It is interesting to note that both articles were developed in countries far from each other - Brazil and India - but in both cases, the need for this harmony is the same.

In the other three studies, effects of high dilutions in animal models are observed, two of them being executed in rodents and one in fish. The observed effects show a broad range of biological implications, ranging from behavioral changes toward adaptation of newborns to environmental oscillations, including proximity to the mather - a very common situation in free-living animals until the growth of malignant tumor by immunomediated mechanisms held in the tumor microenvironment. It is also observed the possibility of biopsical changes in fish brain exposed to alcohol intoxication and its adaptive effects

In all cases, high dilutions act as agents of facilitation to the adaptive process of biological systems, at different metric scales and environmental scenarios, from changes in tumoral microenvironment in a host tissue to the performance in the cultivation of large trees. Undoubtedly, the range of effects of high dilutions and of homeopathy extrapolates the clinical microcosm, inspiring scientists to propose and apply new ways of acting in the world.

Received: December 02, 2018. Accepted: December 04, 2018.

(C) International Journal of High Dilution Research.

Not for commercial purposes. 